\title{
Tuberculosis-related choriocapillaritis (multifocal-serpiginous choroiditis): follow-up and precise monitoring of therapy by indocyanine green angiography
}

\author{
Giulia De Luigi · Alessandro Mantovani • \\ Marina Papadia $\cdot$ Carl P. Herbort
}

Received: 24 August 2011/Accepted: 22 December 2011/Published online: 17 January 2012

(C) Springer Science+Business Media B.V. 2012

\begin{abstract}
To report the case of a patient initially diagnosed with acute posterior multifocal placoid pigment epitheliopathy (APMPPE), characterized by relentless evolution despite high-dose steroid therapy. An interferon-gamma release assay (IGRA) indicated a diagnosis of suspected tuberculous choriocapillaritis and the disease responded only to massive inflammation suppressive therapy and antibiotic therapy. Case report. Review of clinical features and investigational procedures. Smoldering relentless evolution and
\end{abstract}

G. De Luigi · M. Papadia ( $\square)$ · C. P. Herbort ( $\square)$

Centre for Specialized Ophthalmic Care (COS),

6, rue de la Grotte, 1003 Lausanne, Switzerland

e-mail: marinapapadia@yahoo.com

C. P. Herbort

e-mail: carl.herb@bluewin.ch

G. De Luigi

Dipartimento di discipline medico-chirurgiche della comunicazione e del comportamento, Università degli studi di Ferrara, Sezione di clinica oculistica,

Ferrara, Italy

A. Mantovani

Department of Ophthalmology, Ospedale Valduce,

Como, Italy

M. Papadia

Eye Clinic, Department of Neurosciences, Ophthalmology

and Genetics, University of Genova, Genoa, Italy

C. P. Herbort

University of Lausanne, Lausanne, Switzerland subsequent arrest of progression could be precisely monitored by indocyanine green angiography (ICGA). The patient did not recover after standard antitubercolosis (TB) therapy combined with corticosteroid. A fourth antibiotic had to be added in order to stop the progression of the retinal disease. In each case of choriocapillaritis such as APMPPE an infectious cause including TB has to be excluded making IGRA tests unavoidable. As the main structure involved is the choriocapillaris the most precise follow-up or monitoring is obtained with ICGA.

Keywords Multifocal-serpiginous choroiditis . Choriocapillaritis - APMPPE - Tuberculosis ·

ICGA $\cdot$ Antitubercular therapy

\section{Introduction}

Inflammatory diseases of the choriocapillaris (formerly called 'white dot syndromes') are a spectrum of conditions including well-known entities such as acute posterior multifocal placoid pigment epitheliopathy (APMPPE), multiple evanescent white dot syndrome, multifocal choroiditis and serpiginous choroiditis (SC) as well as entities difficult to categorize.

Moreover, clinical presentation of many of these entities frequently overlap rendering exact categorization difficult. In the literature intermediary forms between APMPPE and SC have been described. Some authors called these cases 'ampiginous choroiditis' [1] 
and others called them 'relentless placoid chorioretinitis' [2].

Sometimes we come across cases of choriocapillaritis that do not fall into the well-defined categories cited above. It is of course desirable to classify a case into these known entities but it is not essential. It is much more important with choriocapillaritis to be aware of the disease mechanism (inflammatory choriocapillaris non-perfusion) instead of trying to name the disease. It is also much more important to establish the exact extension of lesions for which indocyanine green angiography (ICGA) is indispensable [3].

Choroidal circulatory status can only be meaningfully assessed by ICGA and this imaging modality is the most suitable to confirm the correct diagnosis. For the follow-up and monitoring of lesions, however, ICGA is unavoidable. Here, we report the case of a patient who was initially diagnosed as suffering from APMPPE and who received high-dose systemic corticosteroids that did not arrest the progression of the disease. Only combined massive anti-tuberculosis (TB) and immunosuppressive therapy that was started after receiving a positive interferon gamma release assay (IGRA) succeeded in stopping the disease progression.

\section{Case report}

A 42-year-old otherwise healthy Caucasian woman presented to the Centre for ophthalmic specialised care (COS) in Lausanne, Switzerland with a 4-month history of halos and decreased vision in the left eye. Fluorescein angiography (FA) performed elsewhere was normal in the right eye (RE), while the left eye (LE) showed patchy early hypofluorescent lesions, mainly located in the posterior pole, with late leakage at their borders and the presence of vasculitis. A diagnosis of APMPPE was put forward by her former physicians and she received systemic corticosteroids (3-day $500 \mathrm{mg}$ intravenous methylprednisolone followed by oral prednisone $50 \mathrm{mg} /$ day for 2 months tapered over 4 months to $6.25 \mathrm{mg} /$ day) and antiviral treatment (valaciclovir $3 \mathrm{~g} /$ day for 3 months tapered over 4 months to $1 \mathrm{~g} /$ day).

At presentation at our center, she was still under systemic corticosteroids ( $6.25 \mathrm{mg} /$ day) and valaciclovir ( 1 g/day). Her visual acuity was $20 / 20$ in the RE and $20 / 80$ in the LE, and intraocular pressure by Goldmann tonometry was $10 \mathrm{mmHg}$ in both eyes.
Anterior segments were normal but a subclinical inflammation in the anterior chamber of the LE was revealed by laser flare photometry $(8.7 \mathrm{ph} / \mathrm{ms}$; normal values 3-6 ph/ms) (Kowa FM-500; Kowa Company Ltd., Electronics and Optics Division, Tokyo, Japan). Vitreous cells were absent. Dilated fundus examination was unremarkable in the RE but the LE showed multiple yellow-white lesions and some pigmented scars (Fig. 1A, B). Visual field examination showed a central relative scotoma in the LE. Optical coherence tomography (OCT) (OTI-Spectral OCT/SLO; Ophthalmic Technologies Inc., Toronto, ON, Canada) was normal in the RE while the LE showed some disruption of the retinal pigment epithelium across the subfoveal area (Fig. 1C, D). Microperimetry was within normal limits in the RE whereas it was pathologic in the LE with a visual score of 302/560 (10.8 dB) (normal range 13-19 dB) (Pattern Polar $3^{\circ}-11^{\circ}$, Size Goldmann III OTI-Spectral OCT/SLO microperimetry; OTI Inc.). We performed another FA which was normal in the RE, while the LE showed new early hypofluorescent lesions with late hyperfluorescence (Fig. 1E, F). ICGA was performed to map out the exact areas of involvement; the RE was unremarkable and the LE showed numerous hypofluorescent lesions of different sizes in the early, intermediate and late frames indicating areas of chorioretinal scars and/or choriocapillaris non-perfusion (Fig. 1G, H). On ICGA, lesions were more clearly visible and more well delineated when compared to lesions on FA.

In addition to the laboratory tests performed at the onset of disease that were within normal limits, we performed a IGRA (QuantiFERON-TB ${ }^{\mathrm{TM}}$ Gold; Cellestis, Victoria, Australia) which was strongly positive $(2.52 \mathrm{IU} / \mathrm{mL}$; cut-off value $0.35 \mathrm{IU} / \mathrm{mL})$ in the setting of a non-vaccinated patient. No intraocular fluid was sampled and polymerase chain reaction was not performed, because these examinations are repeatedly negative in non-endemic areas. An immunemediated choriocapillaritis related to Mycobacterium tuberculosis was suspected. The patient was referred to a general physician in order to search for active foci of extraocular TB which proved negative. A course of three-drug anti-TB therapy was started (ethambutol $15 \mathrm{mg} / \mathrm{kg} /$ day, isoniazid $4 \mathrm{mg} / \mathrm{kg} /$ day, rifampicin $10 \mathrm{mg} / \mathrm{kg} /$ day) and oral steroids were maintained and an immunosuppressant (azathioprine $2.5 \mathrm{mg} / \mathrm{kg}$ / day) was added. As the patient did not tolerate 
Fig. 1 Color fundus photographs at presentation of the right normal eye (A) and left affected eye (B) showing yellowish multifocal lesions surrounded by some pigmented scars. OCT shows interruptions at the level of the retinal pigment epithelium and

inhomogeneous retina (D) as compared to the right non-affected eye (C). Late FA frames $(\mathbf{E}, \mathbf{F})$ show hyperfluorescent lesions. $\operatorname{ICGA}(\mathbf{G}, \mathbf{H})$ shows hypofluorescent areas indicating chorioretinal scars and/or choriocapillaris non-perfusion. There are more lesions than seen on FA. These additional lesions account for new areas of involvement

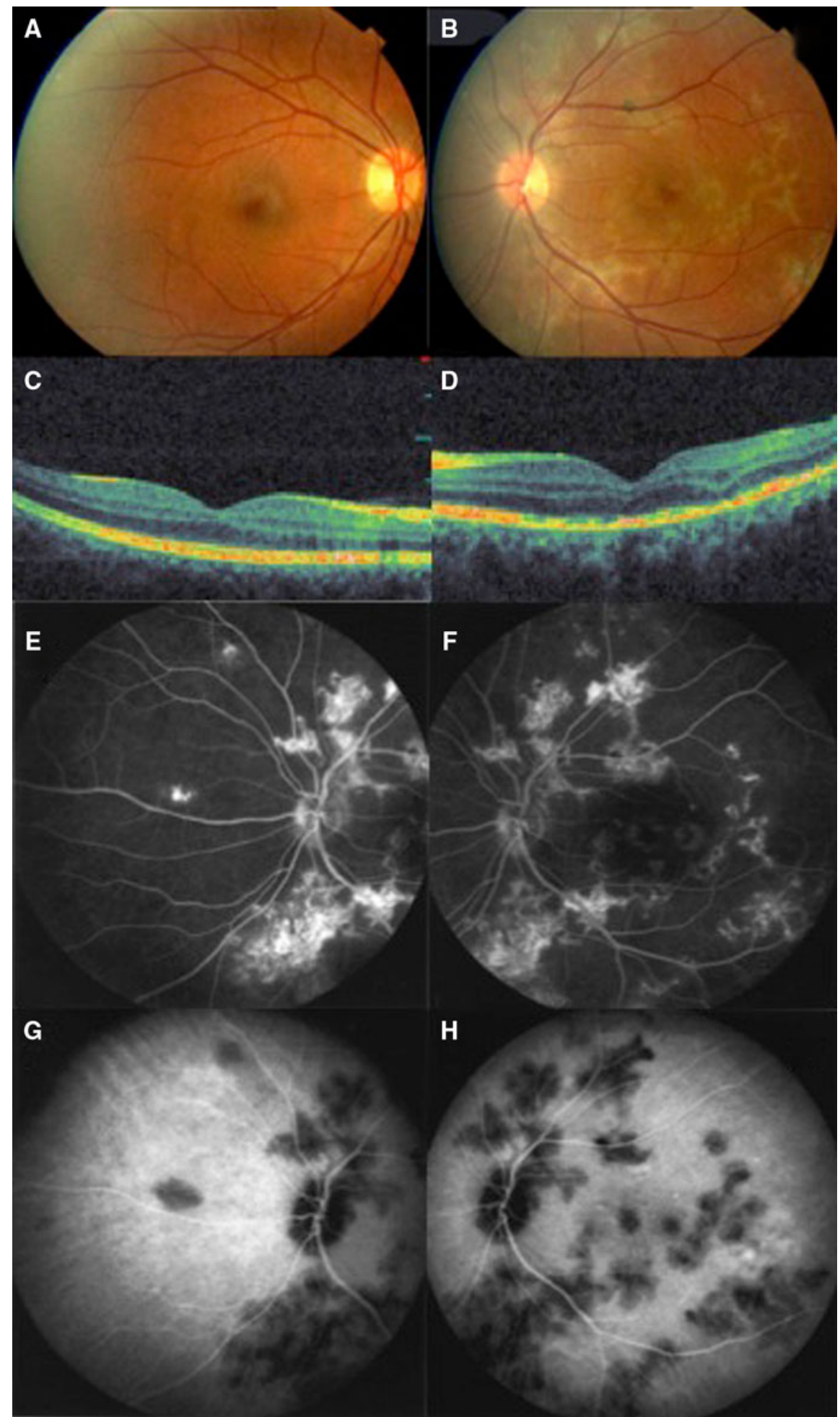


azathioprine because of abdominal pain she was changed to mycophenolic acid (1,440 mg/daily).

Visual acuity in the LE improved to $20 / 20$ in the first 2 months of treatment, but new choroidal lesions appeared on the ICG frames (Fig. 2) and a fourth antiTB drug was added (pyrazinamide $20 \mathrm{mg} / \mathrm{kg} /$ day).

Four months later the visual acuity remained stable in the LE but the ICGA showed new active lesions despite maximal anti-TB therapy (Fig. 2C, D). Therefore, oral steroids were started again (prednisone $0.5 \mathrm{mg} / \mathrm{kg} /$ day).

Two months later, under quadruple anti-TB therapy combined with mycophenolic acid and oral corticosteroid treatment, the patient did not show new lesions for the first time; her visual acuity remained 20/20 in the LE, inflammation in the anterior chamber of LE decreased to $4.7 \mathrm{ph} / \mathrm{ms}$, and the microperimetry increased to $464 / 560(16.6 \mathrm{~dB})$ with almost complete resolution of the central relative scotoma. ICGA findings were still negative for new lesions and the more recent lesions had regressed and were barely visible anymore and not visible at all on FA (Fig. 3). The patient remained with no additional lesions at 9 month follow-up.

\section{Discussion}

This case is of particular interest because of its unilaterality and its progressiveness. Therefore this was an unclassifiable form of choriocapillaritis most probably due to an immune-mediated choriocapillaritis related to TB with clinical features similar to the cases described by Gupta et al. [4]. Although these types of ampiginous cases were believed to be purely immunogenic and normally respond to systemic corticosteroids and/or immunosuppressants, a different form related to TB has been suspected since 1952 [5]. In 2003, Gupta and colleagues found a relationship between TB and conditions resembling APMPPE and SC in patients from endemic TB areas. They called these cases serpiginous-like choroiditis and
Fig. 2 Angiographic frames 2 months after presentation. FA (A, B) shows new lesions (arrows), but ICGA $(\mathbf{C}, \mathbf{D})$ shows new hypofluorescent lesions which are much more widespread (arrows)

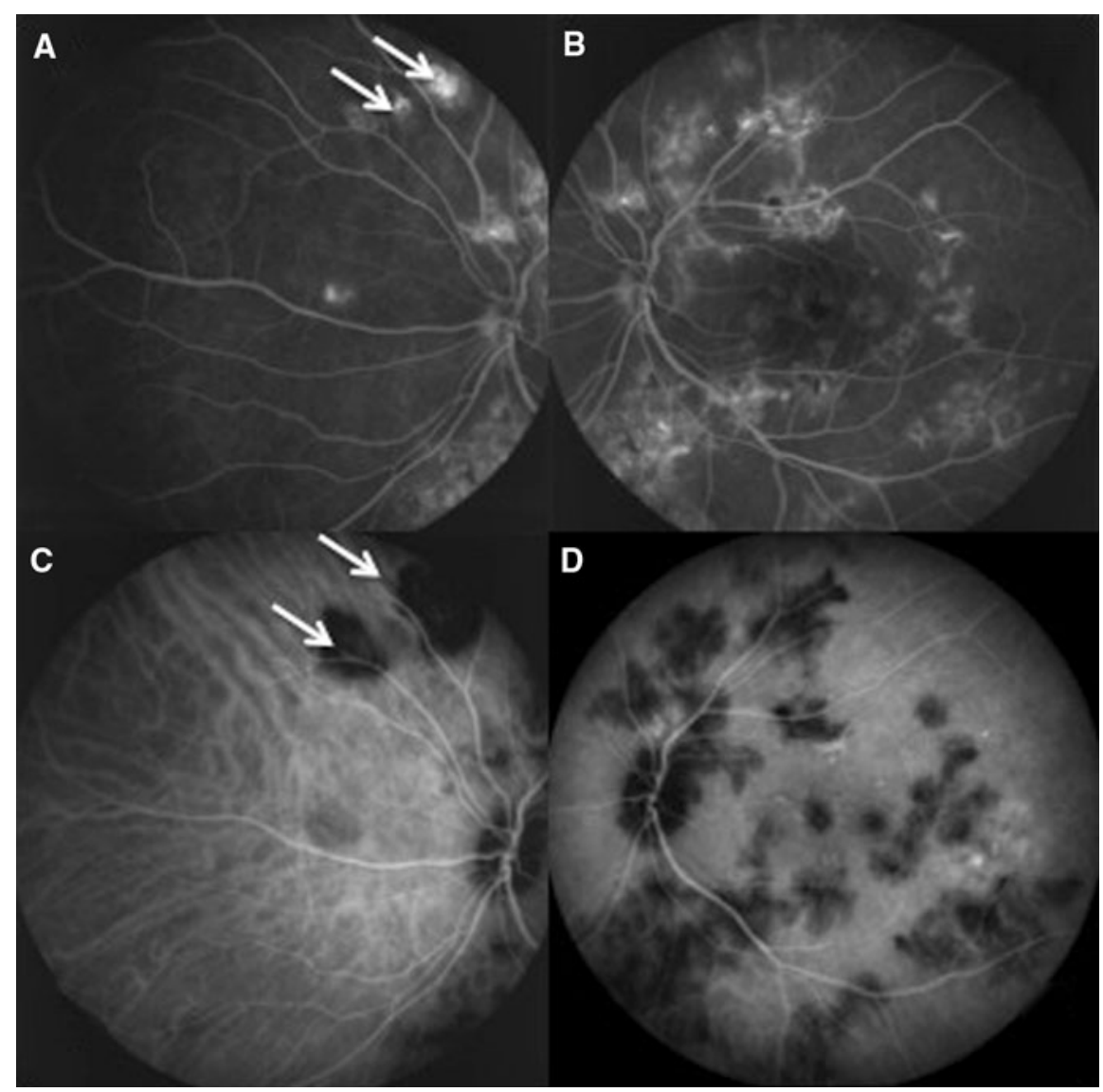


Fig. 3 Angiographic findings in the healed stage. FA (A, B) showing late hyperfluorescence caused by retinal pigment epithelium atrophy (window effect). ICGA $(\mathbf{C}, \mathbf{D})$ shows only cicatricial lesions; the new lesions, well identified in Figs 1 and 2, are barely visible anymore (arrows)

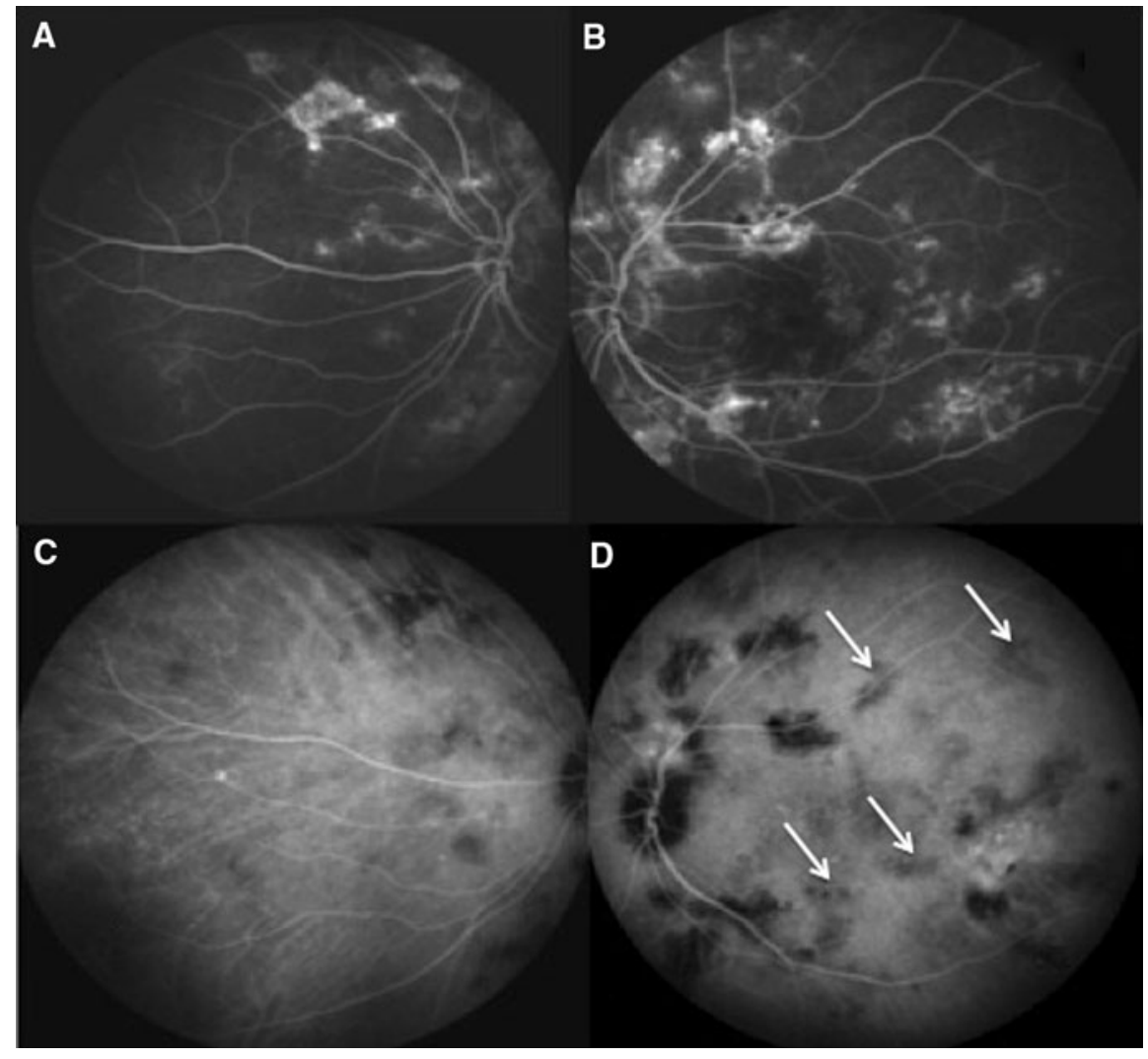

found that the lesions responded to tuberculostatic drugs along with corticosteroids (TB serpiginous-like choroiditis). This terminology was recently revised during the International Ocular Inflammation Society Congress in November 2011, in Goa, India, where the terminology 'multifocal-serpiginous choroiditis' was put forward. In our case report we prefer to talk of 'choriocapillaritis' instead of 'choroiditis' because, thanks to ICGA, it is obvious that the lesion process is at the level of the choriocapillaris. The exact pathogenesis of multifocal-serpiginous choroiditis is unknown; the choroiditis, or better the choriocapillaritis, may represent a hypersensitivity reaction to a minimal load of acid-fast bacteria in the choroid that results in choriocapillaritis with a serpiginous pattern (multifocal-serpiginous choroiditis) [6]. Multifocalserpiginous choroiditis can have two distinct patterns-the classical serpiginous form or multifocal presentation that resembles APMPPE [4] in its first stages which is the case here, or a typical serpiginous pattern or a mixed APMPPE-serpiginous pattern called ampiginous choroiditis in the past; these lesions reveal a relentless progression despite administration of systemic corticosteroids and immunosuppressants. The combination of anti-TB treatment seems to be the only way of stopping progression of recurrences. In our case quadruple anti-TB and immunosuppressive therapy did not completely stop the disease progression which was achieved only when corticosteroids were re-added.

The case presented here is particularly interesting since a TB component was demonstrated in a patient from a non-endemic region. Moreover arrest could be obtained only when optimal combination of inflammation suppressive therapy and anti-TB therapy was reached.

In this case the essential contribution of ICGA is very well demonstrated as it showed many more lesions and increased extension of lesions than seen on FA and their regression showing the impact on therapy.

In conclusion SC is a more morphological diagnosis and should be considered as TB until proven otherwise even in non-endemic countries. Nowadays we have the diagnostic means to rule out TB, using IGRA tests. Immunosuppression alone should only be given once the TB component has been excluded. 


\section{References}

1. Nussenblatt RB, Whitcup MW (1996) Uveitis: fundamentals and clinical practice. Mosby, Philadelphia

2. Jones BE, Jampol LM, Yannuzzi LA, Tittl M, Johnson MW, Han DP, Davis JL, Williams DF (2000) Relentless placoid chorioretinitis: a new entity or an unusual variant of serpiginous chorioretinitis? Arch Ophthalmol 118(7):931-938

3. Herbort CP (2009) Inocyanine green angiography, chap 4. In: Gupta A, Gupta V, Herbort CP, Kharaillah M (eds) Uveitis. Jaypee, New Delhi, p 106
4. Gupta V, Gupta A, Arora S, Bambery P, Dogra MR, Agarwal A (2003) Presumed tubercular serpiginouslike choroiditis: clinical presentations and management. Ophthalmology 110(9):1744-1749

5. Witmer R (1952) A specific form of recidivating choroiditis [in undetermined language]. Ophthalmologica 123(4-5): 353-354

6. Gupta V, Gupta A, Rao NA (2007) Intraocular tuberculosis: an update. Surv Ophthalmol 52(6):561-587 